To appear in Proceedings of 1989 Workshop on Advanced Accelerator

Concepts, Lake Arrowhead, Calif. 9-13 January 1989.

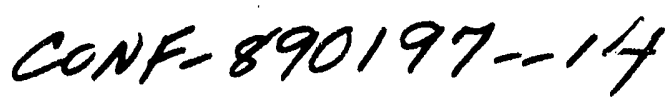

\title{
FOKKER-PLANCK TRANSPORT IN SOLID STATE ACCELERATOR CONCEPTS
}

\author{
B. Newberger and T. Tajima
}

Institute for Fusion Studies and Department of Physics, The University of

Texas at Austin, Austin, Texas 78712

CONF-890197--14

ABSTRACT

DE91 001971

Particle transport in a crystalline solid under channeling conditions is considered by means of a Fokker-Planck description. The model includes electron multiple scattering, radiation damping and an accelerating electric field. Analytic solutions have been obtained using a harmonic potential model to describe the channeling forces. These solutions will be described.

\section{INTRODUCTION}

The phenomenon of the channeling ${ }^{1}$ of energetic charged particles along axes and planes of high symmetry in crystalline solids, has prompted several investigators ${ }^{2-7}$ to suggest schemes for accelerating particles in which the lattice fields would play the part of the magnetic transport system in conventional accelerators. Different schemes would use different methods for obtaining the accelerating gradient. Recent work has suggested an optical field in the $\mathrm{x}$-ray region, ${ }^{2}$ akin to the microwave fields driving conventional accelerators, while another ${ }^{3}$ would exploit the fields of collective modes driven by an external source similar to plasma based accelerators operating at luw'er density. The accelerating gradients can be considerable with $1 \mathrm{GeV} / \mathrm{em}$ suggested in the first case and fields as large as $100 \mathrm{GeV} / \mathrm{cm}$ in the second. Most of these researchers recognized that the multiple scattering would be considerable even though it is reduced by the channeling for positive particles. Some have estimated this, accounting for the $(+)$ effect of the accelerating field on the emittance. ${ }^{3,5}$ In this work, we have examined this problem by means of a Fokker-Planck transport model. Analytic solutions have been obtained for a harmonic channel potential. We will first define the model and then discuss one solution we have found.

\section{FOKKER-PLANCK MODEL}

We have adopted a one-dimensional Fokker-Planck treatment for the distribution function of the particles in the crystal accelerator. In the case of axial channeling, this describes the behavior of the projected distribution function in a radial plane, all of wiich are equivalent in an axisymmetric channel. While this is not necessarily so in general, it should be reasonable in the case of proper channeling. In any case, the qualitative features of the results might well be expected to persist even in more general situations. For planar channeling, the Aco2-88ER40436 
problem is one-dimensional a priori. Furthermore, we make a paraxial approximation $p_{\perp} / p_{z} \ll 1, p_{z} \simeq p \gg 1$ and retain the pitch angle only to lowest order: $\theta \simeq p_{\perp} / p$. Under these assumptions, the Fokker-Planck equation becomes

$$
\begin{aligned}
0= & \frac{1}{p c} \frac{\partial f}{\partial t}+\theta \frac{\partial f}{\partial x}+\frac{\partial f}{\partial z}+\tilde{E} \frac{\partial f}{\partial p}-\frac{\tilde{u}_{x}}{p} \frac{\partial f}{\partial \theta}-\beta \frac{\partial f}{\partial p} \\
& -\beta_{R} \frac{\partial(\theta f)}{\partial \theta}-\frac{1}{2} \frac{\partial}{\partial \theta}\left(\frac{\left(\Delta \theta^{2}\right)}{\Delta t} \frac{\partial f}{\partial \theta}\right) .
\end{aligned}
$$

Momenta are expressed in unitis of the rest momentum of the projectile $m_{I} c$ and energy in units of the rest, energy.

The first five terms arise from the convective derivative in phase space; the fourth and fifth terms due to the accelerating field (taken to be in the $z$-direction) and the channeling potential respectively. The next term accounts for energy losses such as bremsstrahlung or collisional ionization. The remaining terms are due to radiation damping arising from the emission of channeling radiation and multiple scattering. ${ }^{8}$ Under channeling conditions for positive particles, the electrons in the medium dominate the multiple scattering. For our purposes, the situation in which the acceleration gradient exceeds the losses is of interest. A boundary value problem is appropriate and therefore we look for steady-state solutions, $\partial f / \partial t=0$.

Analytic solutions can be readily obtained when $\tilde{u}$ is harmonic. More general cases could be treated by perturbation theory (weak anharmonicity) or numerical solutions based on the method of solution to now be brielly described. The procedure is to introduce the characteristics of the first order operator in Eq. (1). (The term $-\beta_{R} f$ can be handled trivially by introducing $\chi=e^{-\beta_{R} z} f$. This has no effect on the statistical averages.) The general solution of inis coupled set of ODE's contains a set of integration constants. These, through the solution of the ODE's define a transformation of variables from $(z, \theta, x, p) \rightarrow(s, \eta, \zeta, \xi)$. Applying this to Eq. (1) casts it in a form to which a lemma of Chandrasekhar ${ }^{3}$ applies. The Green's function solution of Eq. (1) can then be written down immediately. For a given distribution of particles at $z=0$, the distribution at interior points $z>0$ is given by a convolution. Moments over the distribution as functions of $z$ can be obtained in a straightforward way.

\section{STRONG ACCELERATION RADIATION-FREE SOLUTION}

The case of strong acceleration (acceleration $>$ losses) is of interest. For heavy particles $\left(m \geq m_{p}\right.$ ), the radiation is negligible (Ultimately it would be very nice to get into the radiative regime for them, too.) The solution of the characteristic equations can be expressed in termr of Bessel functions. The calculation of the moments can be done analytically. First we observe that

$$
\tilde{u}=\frac{k x^{2}}{2}=\frac{v_{0} x^{2}}{2 m_{I} c^{2}}
$$


where $v_{0}$ is the channel well curvature. It is typically $\sim$ few $\times 10^{16} \mathrm{ev} / \mathrm{cm}^{2}$. Even for an accelerating gradient of $100 \mathrm{GeV} / \mathrm{cm}, k / \alpha \gg 1$. The appropriate asymptotic expansions of the Bessel lunctions can be used. We find

$$
\begin{aligned}
& \left\langle x^{2}\right\rangle \simeq \frac{D}{\alpha k}\left[\left(\frac{\alpha z+p_{0}}{p_{0}}\right)^{1 / 2}-1\right] \\
& \left\langle\theta^{2}\right\rangle \simeq \frac{D}{\alpha\left(\alpha z+p_{0}\right)}\left[\left(\frac{\alpha z+p_{0}}{p_{0}}\right)^{1 / 2}-1\right] \\
& \langle x \theta\rangle=0,
\end{aligned}
$$

where $p_{0}$ is the initial energy and $D \equiv \frac{p^{2}}{2}\left\langle\frac{\Delta \theta^{2}}{\Delta z}\right\rangle$.

Note that in the absence of acceleration, $\alpha=0$, the resuit just reduces to a random walk. The (unnormalized rms emittance, $\epsilon$, given by ${ }^{10}$

$$
\begin{aligned}
& \epsilon=4\left[\left\langle x^{2}\right\rangle\left\langle\theta^{2}\right\rangle-\langle x \theta\rangle^{2}\right]^{1 / 2} \quad \text { is then } \\
& \epsilon \simeq \frac{4 D}{\alpha \sqrt{k}} \frac{1}{\left(\alpha z+p_{0}\right)^{1 / 2}}\left[\left(\frac{\alpha z+p_{0}}{p_{0}}\right)^{1 / 2}-1\right] .
\end{aligned}
$$

In the limit $\alpha z \gg p_{0}$, this becomes $\epsilon \simeq 4 D / \alpha \sqrt{k p_{0}}$, constant. This is consistent with the estimates in Ref. 3. Using the expression in Ref. 8 for the multiple scattering coefficient, we have

$$
D=2 \pi\left(\frac{1}{137}\right)^{4} a_{0}^{2} N Z_{\text {val }}\left(\frac{m_{\mathrm{e}}}{m_{I}}\right)^{2} L_{R}
$$

where $L_{R} \simeq 10, a_{0}=$ Bohr radius $=.53 \times 10^{-8} \mathrm{~cm}, N=$ electron number density $\approx 10^{22} / \mathrm{cm}^{3}$ and $Z_{\mathrm{val}}=$ no. of valence electrons/atom. For positrons, $D \simeq 50 / \mathrm{cm}$. Note that logarithmic energy dependencies in fundamental processes have been neglected throughout.

Recall that the solutions given here are for the Green's function solution. If a different initial distribution is specified, the moments are modified accordingly.

As a numerical example consider protons at an initial energy of $1 \mathrm{TeV}$, $p_{0}=1.1 \times 10^{3}$. With a channel potential of about $50 \mathrm{eV}$, which is rather typical, ${ }^{1}$ $v_{0} \simeq 2 \times 10^{16} \mathrm{eV} / \mathrm{cm}^{2}$ for typical channel radii of $4 \times 10^{-8} \mathrm{~cm}$. This gives the following parameters:

An accelerating gradient of $100 \mathrm{GeV} / \mathrm{cm}$

$$
\begin{aligned}
\Rightarrow \alpha & =1.1 \times 10^{2} / \mathrm{cm} \\
k & =v_{0} / m_{I} c^{2} \simeq 2.2 \times 10^{7} / \mathrm{cm}^{2} \\
& E_{f}=100 \mathrm{TeV} \Rightarrow z=10^{3} \mathrm{~cm}
\end{aligned}
$$


Then Eq. (6) yields a final emittance value of

$$
\epsilon \simeq 5 \times 10^{-12} \mathrm{rad}-\mathrm{cm} .
$$

This is larger than the channel acceptance which would be obtained from the expressions in Ref. 3. Of course, when the emittance is comparable to the channel acceptance, the simple form adopted for the channel well is suspect, although it would seem that estimates in this case would be too optimistic. Nevertheless, the qualitative behavior is consistent with other work. It is clear that means to reduce the multiple scattering could be of some benefit to improve the error margin. Some possibilities for this will be discussed elsewhere. ${ }^{10}$

\section{SUMMARY AND CONCLUSIONS}

We have considered the transport of charged particles under channeling conditions in crystal accelerator schemes using a Fokker-Planck model. Analytic solutions have been obtained. These can be used to find the statistical properties of the particle bearn. Solutions, given any specified distribution at the boundary, can be obtained by convolution. As an example, we have considered the emittance growth of a $\delta$-fur.ction beam of protons as it is accelerated under channeling conditions. Extensions of the work to include the effect of radiation damping which is important for light particles (positrons and muons) and to channeling in bent crystals is in progress. The latter is important in the application to beam steering in accelerators. 


\section{ACKNOWLEDGMENTS}

We would like to thank Dr. F.R. Huson, Dr. W.W. Mackay, and Dr. S. Ohnuma for many helpful discussions.

This work was supported by the Texas Accelerator Center.

\section{REFERENCES}

1. Donald S. Gemmell, Rev. Mod. Phys. 46, 129 (1974).

2. T. Tajima and M. Cavenago, Phys. Rev. Lett. 59, 1440 (1987).

3. P. Chen and R.J. Noble, in Relativistic Channeling; edited by Richard A. Carrigan, Jr. and James A. Ellison (Plenum, New York, 1987) pp. 517522.

4. A.F. Pisarev, Zh. Tekh. Fiz. 49, 786 (1979) [Sov. Phys. Tech. Phys. 24, 456 (1979)].

5. V.V. Beloshitsky and M.A. Kumakhov, Jokl. Akad. Nauk SSSR 249, 100 (1979) [Sov. Phys. Dokl. 24, 916 (1979)].

6. I.A. Grishaev and N.N. Nasonov, Pis'ma Zh. Tekh. Fiz. 3, 1084 (1977) [Sov. Tech. Phys. Lett. 3, 446 (1977)].

7. Alvin Kanofsky, Rev. Sci. Inst. 48, 34 (1977).

8. M.A. Kumakov and R. Wedell, Phys. Stat. Sol. (h) 92, 65 (1979).

9. Claude Lejeune and Jean Aubert, in Applied Charged Particle Optics, Part A, edited by A. Septier (Academic, New York, 1980) Fp. 159-259.

10. B. Newberger, T. Tajima, F.R. Huson, W. Mackay, B.C. Covington, J.R. Payne, Z.G. Zou, N.K. Mahale, and S. Ohnuma, Proceedings 1989 Particle Accelerator Conference, IEEE Conference Record 89CH2669-0, March 1989, : s àppear.

\section{DISCLAIMER}

This report was prepared as an account of wrrk sponsored by an agency of the United States Government. Neither the Unites States Government nor any agency thereof, nor any of their employees, makes any warranty, express or iraplied, or assumes any legal liability or responsibility for the accuracy, completeness, or usefulness of any information, apparatus, product, or process disclosed, or represents that its use would not infringe privaiely owned rights. Reference herein to any specific commercial product, process, or service by trade name, trademark, manufacturer, or otherwise does not necessarily constitute or imply its endorsement, recom. mendation, or favoring by the United States Government or any agency thereof. The views and opinions of authors expressed herein do not iecessarily state or reflect those of the United States Government or any agency thereof. 

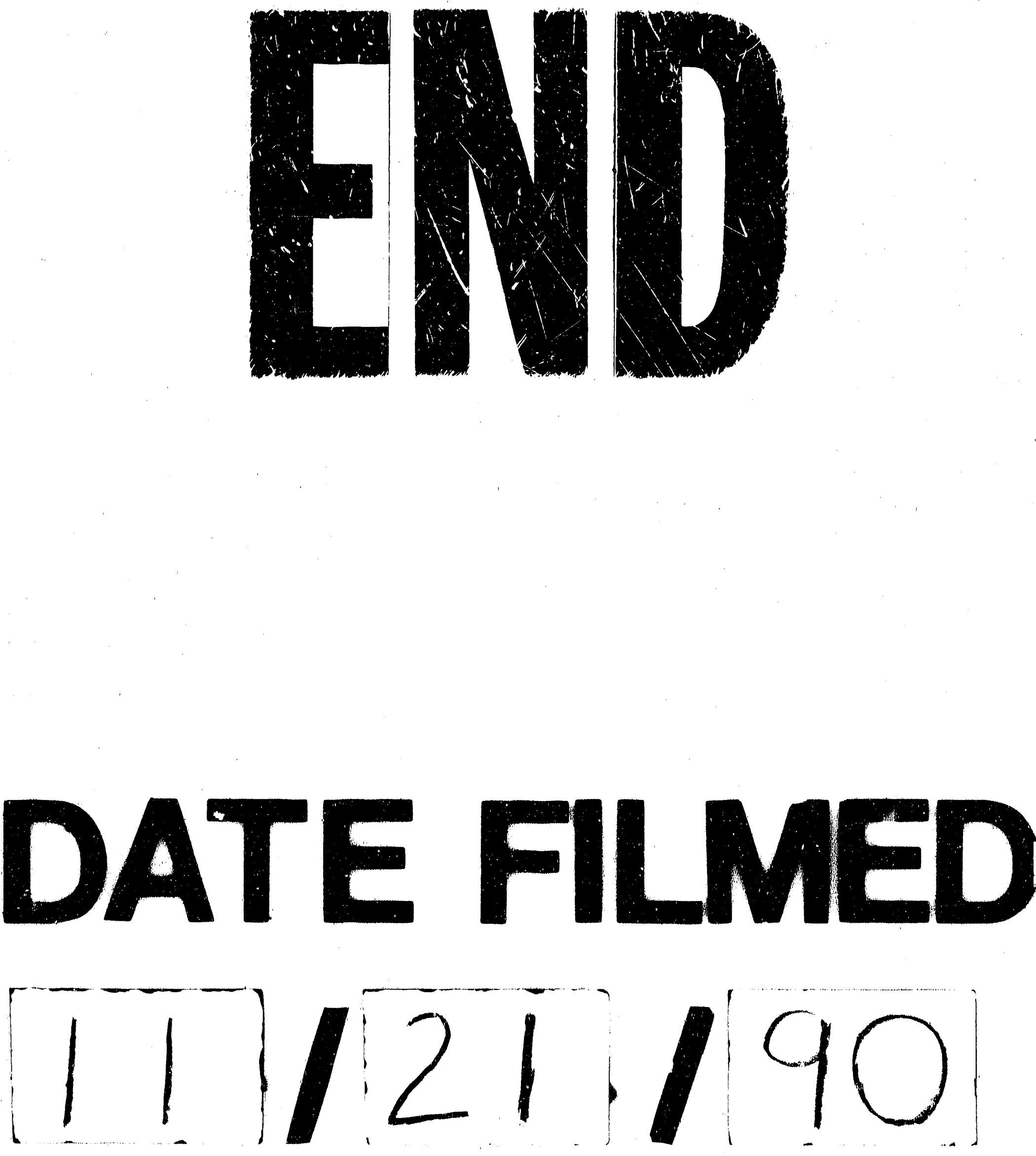
